\title{
Missbrauch der Psychiatrie
}

\author{
Abuse of Psychiatry
}

Autor

Institut
Tilman Steinert

Zentrum für Psychiatrie Südwürttemberg, Ravensburg-Weissenau

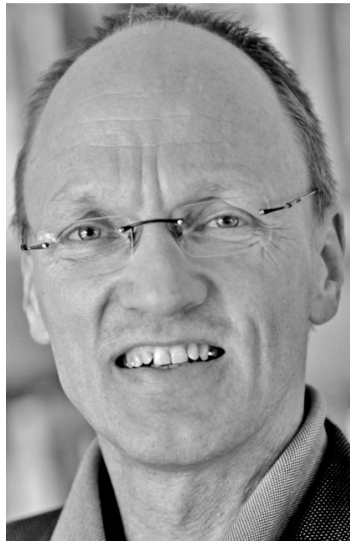

Tilman Steinert
Bibliografie

DOI http://dx.doi.org/

10.1055/s-0034-1370079

Psychiat Prax 2014; 41 :

237-238

(c) Georg Thieme Verlag KG

Stuttgart · New York

ISSN 0303-4259

Korrespondenzadresse

Prof. Dr. med. Tilman Steinert

Zentrum für Psychiatrie

Südwürttemberg

Weingartshoferstraße 2

88214 Ravensburg-Weissenau

tilman.steinert@zfp-zentrum.de
Missbrauch der Psychiatrie scheint auf den ersten Blick ein historisches und nichtwestliches Thema zu sein. Historisch war der Missbrauch der Psychiatrie immer der Missbrauch einer medizinischen Disziplin für staatliche Ordnungsinteressen. Die in beträchtlichem Maße von philanthropischen Motiven und staatlichen Investitionen getragene Gründung der psychiatrischen Anstalten zu Beginn des 19. Jahrhunderts wurde schon von Anbeginn von der Überlegung begleitet, dort auch störende und dissoziale Personen einer Einrichtung der öffentlichen Ordnung zuweisen zu können. Ob das Heilungs- oder das Ordnungsmotiv führend war, ist unter Psychiatriehistorikern umstritten [1 -3]. In der zweiten Hälfte des 19. Jahrhunderts bekannten sich führende Psychiater wie Kraepelin offen dazu, dass die Anstaltsasylierung bei weitgehend fehlenden therapeutischen Möglichkeiten den Betroffenen sogar schädlich sein könne, in erster Linie aber dazu diene, sie von der Fortpflanzung abzuhalten [4]. Eine therapeutische Blüte erlebte die Psychiatrie nach den folterähnlichen Methoden in der ersten Hälfte des 19. Jahrhunderts erst wieder mit der Militärpsychiatrie des Ersten Weltkriegs, die den deutschen Psychiatern allerdings wegen ihrer Methoden und ihrer Ziele den berühmt gewordenen Vorwurf von Sigmund Freund eintrug, sie seien die „Maschinengewehre hinder der Front“ - die darauf abzielten, die Soldaten um jeden Preis wieder „kriegsverwendungsfähig“ zu machen. Der bekannteste und schwerwiegendste Missbrauch der Psychiatrie ereignete sich unter der NS-Diktatur, als führende Psychiater zu Komplizen des Regimes wurden in der Zielsetzung, die Personengruppe der psychisch Kranken systematisch erst von der Fortpflanzung auszuschließen und dann zu ermorden und dabei Methoden der industriellen Massentötung zu entwickeln. Nach dem Zweiten Weltkrieg ist ein Missbrauch der Psychiatrie insbesondere aus der Sowjetunion bekannt geworden. In der DDR gab es historischen Erkenntnissen zufolge keinen systematischen Missbrauch durch den Staat, wohl aber punktuelle Übergriffe etwa mit Verletzungen der Schweigepflicht durch ärztliche Mitarbeiter im Dienste der Staatssicherheit [5]. Gibt es auch einen Missbrauch der Psychiatrie heute, in Mitteleuropa? Aus meinen beruflichen Erfahrungen würde ich dies unbedingt bejahen und halte es, nicht zuletzt aus den genannten historischen Gründen, für eine elementare Aufgabe unseres Berufs, dies zu benennen und uns dagegen zu wehren. Um mit dem Harmlosesten anzufangen - es gibt einen Missbrauch der Psychiatrie durch Patienten. Dass Menschen sich unter dem Vorwand von Suizidgedanken in eine Klinik einweisen lassen und dort ein Attest begehren, dass sie an einer Gerichtsverhandlung zwei Tage später nicht teilnehmen könnten, kommt vor. Auch, dass die Zuweisung in eine psychiatrische Klinik dazu missbraucht wird, externalisierende Verhaltensextreme jeder Art einmal ungezügelt auszuleben. Quantitativ ist dies, wie wir wissen, unbedeutend. Dasselbe gilt für die Fälle eines Missbrauchs durch Angehörige, die die demenzkranke Mutter in der Klinik abliefern und telefonisch nicht mehr erreichbar sind, weil sie in Urlaub gefahren sind. Ärgerlicher, weil häufiger, sind schon die Fälle für den diensthabenden Arzt, in denen psychisch auffällige Personen von Notfallambulanzen ungeachtet ihrer akuten somatischen Beschwerden in die psychiatrische Klinik verlegt werden - weil sie eben schon einmal dort waren, einmal Psychiatrie, immer Psychiatrie. Oder die Fälle, in denen die Verlegung in die psychiatrische Klinik damit begründet wird, der Patient sei auf einer somatischen Station „nicht führbar“, ungeachtet einer diagnostischen Klärung und Indikationsstellung. Noch problematischer ist die vielerorts bekannte Praxis der psychiatrischen Klinik als „Strafkolonie“ - Menschen aus Heimeinrichtungen jeder Art werden bei Verhaltensauffälligkeiten (meist aggressiver Natur) de facto aus disziplinarischen Gründen, oft sogar explizit so be- 
nannt, vorübergehend in eine psychiatrische Klinik verlegt. Da soll die psychiatrische Klinik als Kurzzeitvollzugseinrichtung für Menschen mit einer Behinderung dienen, unter Missbrauch ihrer Funktion zur Krankenbehandlung, unter Berufung auf lange Traditionen und, zugegebenermaßen, bei oft wenig verfügbaren Alternativen. Auch dass Psychiater und in psychiatrischen Einrichtungen Beschäftigte die Psychiatrie missbrauchen, um unter dem Vorwand therapeutischer Notwendigkeiten, in Wirklichkeit aus Motiven eigener Sicherheitsbedürfnisse oder Bequemlichkeiten, Macht auszuüben, wo mehr Freiheit möglich wäre, kommt selbstverständlich vor.

All dies sind nicht die Fälle, die einen Psychiater bewegen, ein Editorial zu schreiben. Dies sind vielmehr gesellschaftliche Phänomene, die beinhalten, dass Teile von Politik und Öffentlichkeit einen Konsens gefunden haben, die Psychiatrie zu bestimmten Zwecken missbrauchen zu wollen. Da ist zunächst einmal der Missbrauch der Psychiatrie (die, wie man immer wieder einmal betonen muss, ein medizinisches Fachgebiet und keine Institution ist, was aber im Sprachgebrauch merkwürdig verschwimmt) als Projektionsfläche für Vorurteile. Diesen Missbrauch betreiben Journalisten ebenso wie Politiker ausgesprochen gerne. Er reicht vom bekannten Missbrauch des Begriffs Schizophrenie als Metapher [6] für alles, was widersprüchlich erscheint bis zu diffamierenden Äußerungen von Politikern übereinander, so etwa CSUGeneralsekretär Scheuer im März 2014 über den ehemaligen SPD-Kollegen Edathy („... gehört in die Klapse“). Sprachlich und kulturell wesentlich elaborierter, gleichwohl ein antipsychiatrisches Klischee bedienend, formulierte Heribert Prantl, Chefredakteur der Süddeutschen Zeitung 2013, dass zur Einweisung in die forensische Psychiatrie, welche „schlimmer als Gefängnis“ sei, „strafrechtlicher Pipifax“ genüge. Wobei im Fall Mollath in keiner seriösen Nachrichtensendung bei der Erwähnung des Worts „Psychiatrie“ die eingeblendeten Fixierbetten fehlten.

Damit nicht genug. Nach den wegweisenden Entscheidungen des Bundesverfassungsgerichts von 2011, die von der DGPPN ausdrücklich begrüßt wurden [7], stehen nun die fälligen Revisionen der Unterbringungsgesetze bzw. Psych-KGs in den Bundesländern an. Im Gesetzentwurf des Bundeslandes Sachsen werden unter $\S 31$ (Sicherungsmaßnahmen) bemerkenswerterweise nun die folgenden Sicherungsmaßnahmen ergänzt: die zeitweise Fixierung und „die medikamentöse Ruhigstellung, die einer zeitweisen mechanischen Fixierung in ihrem Zweck und ihren Auswirkungen gleichkommt“. Notabene, Ärzte sollen Medikamente nicht zur Behandlung, sondern zur „Ruhigstellung“ als Sicherungsmaßnahme verordnen. Die Musterberufsordnung für die in Deutschland tätigen Ärztinnen und Ärzte, die zweifellos auch für Psychiater gilt, formuliert dagegen: „... haben dabei ihr ärztliches Handeln am Wohl der Patientinnen und Patienten auszurichten. Insbesondere dürfen sie nicht das Interesse Dritter über das Wohl der Patientinnen und Patienten stellen“ [8]. Der Staat kann und darf Ärzte nicht zu Aufgaben legitimieren bzw. ihnen diese sogar zuschreiben, die ihnen von der Berufsordnung her eindeutig verboten sind. Es gibt bekanntlich keine Medikamente, die für die Indikation einer Ruhigstellung als Sicherungsmaßnahme zugelassen wären, ebenso wenig eine Differenzierung von Medikamentenverordnungen zur Behandlung und zur „Ruhigstellung“. Behandlung bei krankheitsbedingter Fremdgefährdung gehört zu unseren Aufgaben, eine Ruhigstellung unabhängig von einer Behandlungsindikation aber sicher nicht. Ein weiterer derartiger Prüfstein blieb uns in Deutschland seitens der Politik vorenthalten, die britischen Psychiater haben ihn auf eigenes Drängen erhalten und die niederländischen Kollegen werden bald nachziehen: die ambulante Zwangsbehandlung. Ob sie überhaupt wirksam ist, ist sehr umstritten [9]. Aber die Fokussierung auf die evidenzbasierte Wirksamkeit ist eine gefährliche Verengung: Was bedeutet es für die Bürgerrechte, wenn man damit beginnt, Personen, die keine Straftat begangen haben, von Staats wegen einem präventiven Gesundheits- und Gefährlichkeitsmanagement mit erheblichen Eingriffen in die Freiheitsrechte zu unterziehen, und zwar ungeachtet ihrer Einwilligungsfähigkeit? Dass letztere bei Patienten mit Psychosen, die in den genannten Ländern einer Behandlungsauflage unterworfen werden, dauerhaft aufgehoben ist, dürfte kaum anzunehmen sein. Die deutsche Psychiatrie bekam stattdessen die von der Öffentlichkeit weitgehend unbemerkte „Lösung“ des vom Verfassungsgericht angemahnten Problems der nachträglichen Sicherungsverwahrung mit der Überstellung von Menschen zur Dauerverwahrung, die zwar als gefährlich gelten, bislang aber nicht als psychisch krank gegolten hatten. Die Versuche, diesen Missbrauch forensischpsychiatrischer Behandlungseinrichtungen zu verhindern, waren nur teilweise erfolgreich.

Auch unabhängig davon verbleibt der mir immer wieder verstörend erscheinende Umstand, dass wir zuweilen in der forensischen Psychiatrie insbesondere unter den §64-Patienten solche haben, die ganz offensichtlich sehr viel mehr gefährlich als krank sind und massive Sicherungsmaßnahmen in unseren Kliniken erfordern, während sie z. B. auf ihre Rückkehr in Haft warten. Wenn Sicherungsmaßnahmen und Therapie über längere Zeit in einem offensichtlichen Missverhältnis stehen, muss sich die Frage aufdrängen, ob es sich dabei noch um typische Aufgaben von Ärztinnen und Ärzten, Gesundheits- und Krankenpflegerinnen und -pflegern und Krankenhäusern handelt. Der Justiz, der Politik und der Öffentlichkeit genügt das Etikett, dass diese Menschen angeblich alle „Therapie“ benötigen und erhalten, zur moralischen Beruhigung von eigenen Ängsten und potenziellen Schuldgefühlen. Nicht, dass ich dafür wäre, Therapie vorzuenthalten. Aber die Therapie kann auch zu Menschen kommen, die dies wünschen und deren Sicherung nicht Krankenhäusern übertragen wurde. Wenn wir uns nicht gegen die vielfältigen Aufgabenzuschreibungen wehren, bei denen die Psychiatrie in einem breiten, freilich stets zur Anklage bereiten Konsens von Politik, Öffentlichkeit und somatischer Medizin als Kontrollinstrument missbraucht wird, werden wir das Odium des Zwangs und der Ausübung von Gewalt als Disziplinierung auch immer anhaften haben.

\section{Literatur}

1 Dörner K. Bürger und Irre. Frankfurt/Main: Europäische Verlagsanstalt; 1967

2 Foucault M. Wahnsinn und Gesellschaft. Frankfurt/Main: Suhrkamp; 1969

3 Blasius D. Der verwaltete Wahnsinn. Eine Sozialgeschichte des Irrenhauses. Frankfurt/Main: Fischer; 1980

4 Kraepelin E. Die psychiatrischen Aufgaben des Staates. Jena: Fischer; 1900

5 Süß S. Politisch mißbraucht? Psychiatrie und Staatssicherheit in der DDR. Berlin: Links Verlag; 1998

6 Hoffmann-Richter U. Psychiatrie in der Zeitung. Urteile und Vorurteile. Bonn: Psychiatrie-Verlag; 2000

7 DGPPN, Hrsg. Memorandum der DGPPN zur Autonomie und Selbstbestimmung von Menschen mit psychischen Störungen. Nervenarzt 2012; 83: 1491 - 1493

8 Bundesärztekammer, Hrsg. Berufsordnung für die in Deutschland tätigen Ärztinnen und Ärzte in der Fassung der Beschlüsse des 114. Deutschen Ärztetages 2011 in Kiel. Im Internet: www.bundesaerztekam mer.de/downloads/MBO_08_20112.pdf (Stand: 15.4.2014)

9 Burns T, Rugkåsa J, Molodynski A et al. Community treatment orders for patients with psychosis (OCTET): A randomised controlled trial. Lancet 2013; 381: 1627-1633 